\title{
The origin of pattern information of an apparently moving object during stroboscopic motion
}

\author{
M. W. von GRÜNAU \\ Queen's University, Kingston, Ontario K7L 3N6, Canada
}

\begin{abstract}
The differential contribution of the two flashed stimuli to pattern aspects of the apparently moving object during stroboscopic motion is examined. It is found that metacontrast masking of one of the stimuli abolishes that stimulus' influence on pattern completely, while not interfering with motion information of the same stimulus. Thus, good motion still occurs, but the apparently moving object has pattern characteristics of the unmasked stimulus only.
\end{abstract}

Recently, much evidence has accumulated, based both on neurophysiological experiments with cats and monkeys (Cleland, Dubin, \& Levick, 1971; Cleland, Levick, \& Sanderson, 1973; Dow, 1974; Enroth-Cugell \& Robson, 1966; Fukuda \& Stone, 1974; Ikeda \& Wright, 1974, 1975; Singer \& Bedworth, 1973) as well as on psychophysical experiments with human observers (Kulikowski, 1978a, 1978b; Kulikowski \& Tolhurst, 1974; Tolhurst, 1973; von Grünau, 1978b), suggesting a subdivision of the mammalian visual system: One subsystem seems to be specialized for the processing of pattern or form information and another for the processing of motion information. These systems are also referred to as "sustained" and "transient" systems or channels, a distinction based on certain properties of visual neurons. Despite striking similarities in many aspects between the psychophysical and the electrophysiological classifications, the exact nature of this correspondence is still unknown, and recent criticism casts some doubt on the existence of a straightforward relationship (Lennie, 1980).

Most visual stimuli are capable of exciting both systems, according to the electrophysiological findings. Thus, presentation of any visual stimulus should provide some information leading to the perception of pattern or form and some information leading to the perception of motion. It has been shown that these aspects of visual stimuli can be manipulated independently. Thus, von Grünau (1978a) has shown that in a stroboscopic motion situation, in which the sequential flashing of two spatially separated stimuli gives rise to the perception of a moving object (Wertheimer, 1912), pattern information of one of the stimuli can be interfered with

This research was supported by NSERC Grant A0044. I would like to thank P. C. Dodwell for his most generous support and critical reading of this paper, and Lee Paterson, who served as observer and experimenter. by metacontrast masking without interfering with its motion information.

Since, in these experiments, both stimuli consisted of identical disks, it was not possible to determine how much each of the stimuli was actually contributing to the pattern or form of the apparently moving object. In the present experiments, an attempt was made to assess the contribution of each stimulus by measuring the change in orientation of an apparently moving object when one of two different stimuli was also metacontrast masked. The results indicate that, for the stimuli used (a vertical and a horizontal bar), the masked stimulus does not, indeed, contribute to the perceived orientation, but, at the same time, it does contribute motion information.

\section{METHOD}

\section{Apparatus and Stimuli}

The two stimuli and the mask were presented monocularly in a three-channel tachistoscope with an addition that allowed the presentation of a continuous background, a fixation point, and a comparison stimulus that was adjustable in orientation and position (see Figure 1A).

In Figure 1B, the stimulus configuration is presented as seen by the observer. The first and the second stimuli consisted of a vertical and a horizontal bar, respectively, and were presented for $20 \mathrm{msec}$ with a luminance of $8.6 \mathrm{~cd} / \mathrm{m}^{2}$. The mask consisted of two bars, one on each of the long sides of the masked stimulus bar, and was presented for $120 \mathrm{msec}$ with a luminance of $14.4 \mathrm{~cd} / \mathrm{m}^{2}$. The fixation point (FP) to the left of the first stimulus was a dark spot on the continuously illuminated background field of $3.8 \mathrm{~cd} / \mathrm{m}^{2}$. The comparison stimulus could be moved continuously from a position directly below the first stimulus to a position directly below the second stimulus. Its orientation could be adjusted from -20 to $110 \mathrm{deg}$, with $0 \mathrm{deg}$ corresponding to horizontal and 90 deg to vertical.

\section{Procedure}

The likelihood of seeing motion was measured as a function of the time interval between the onsets of the two stimulus flashes (SOA). This was done for motion between the two bars, between the mask in its leftward position and the right bar, and between the left bar and the mask in its rightward position. The latter two were control conditions. Between the offset of the 



COMPARISON STIMULUS

Figure 1. (A) Three-channel tachistoscope for the presentation of stimuli. A special addition allowed the presentation of a homogeneous background field with a fixation point and a comparison stimulus that could be moved laterally and rotated through 130 deg. (B) Stimulus display as seen by the observer. Dimensions are in degrees of visual angle at a distance of $56 \mathrm{~cm}$.

second flash and the reappearance of the first flash, there was a pause of $2 \mathrm{sec}$, so that motion was seen from left to right only. During the pause, the observer responded "yes" or "no" by pressing the appropriate key. The task was to decide whether smooth continuous motion of one object occurred across the whole space between the two end positions. Fifty responses were recorded at each SOA value within each of the conditions.

A pilot study was conducted in order to assess the effectiveness of masking under the masking condition. The masked stimulus was changed to a bar with a pointed tip that could point either up or down (left or right, in case of the horizontal stimulus). The two possibilities were presented in a haphazard way, and the observer had to correctly identify the direction of the tip. This was done as a function of the time interval between the onsets of the bar and the mask [SOA(masking)]. Again, there was a pause of $2 \mathrm{sec}$ between the end of the mask and the reappearance of the bar, during which the observer responded by pressing the appropriate key. For Experiment 1, the first (left) stimulus was masked, and, in Experiment 2, the second (right) stimulus was masked. Fifty responses were recorded at each SOA value.

To measure the orientation of the apparently moving object along the path, the observer adjusted the orientation of the comparison stimulus, which was positioned haphazardly by the experimenter at one of the following positions: Below the first stimulus (L), below and one-fourth of the way between the first and the second stimulus (1/4), below and halfway between the two stimuli $(1 / 2)$, below and three-fourths of the way between the two stimuli $(3 / 4)$, and below the second stimulus (R). These measurements were made only for the optimal SOA values for motion and/or masking. Twenty adjustments were made at each position.
Before the beginning of a session, the observer was darkadapted for $20 \mathrm{~min}$. The various SOA values (for motion and masking) and the various positions (for orientation) were presented in randomized order.

The two observers (L.P. and M.G.) had normal color and spatial vision, as assessed with the H-R-R Pseudoisochromatic Plates and the Bausch \& Lomb Orthorater, and were well acquainted with psychophysical experiments. Both were well practiced in the tasks of these experiments, and L.P. was unaware of the purpose of the present study.

\section{RESULTS}

\section{Experiment 1: Masking of the First Stimulus}

In this experiment, the optimal SOA for masking of the first (left) bar by the mask was determined for each observer. These values were $20 \mathrm{msec}$ for L.P. and $60 \mathrm{msec}$ for M.G. The likelihood of seeing good motion was then measured for motion between the two bars and for motion between the bars when the first bar was also optimally masked. These results are shown in the upper part of Figure 2 for values of SOA (S1, S2) between the two bars that gave optimal motion (100 msec for L.P., $110 \mathrm{msec}$ for M.G.).

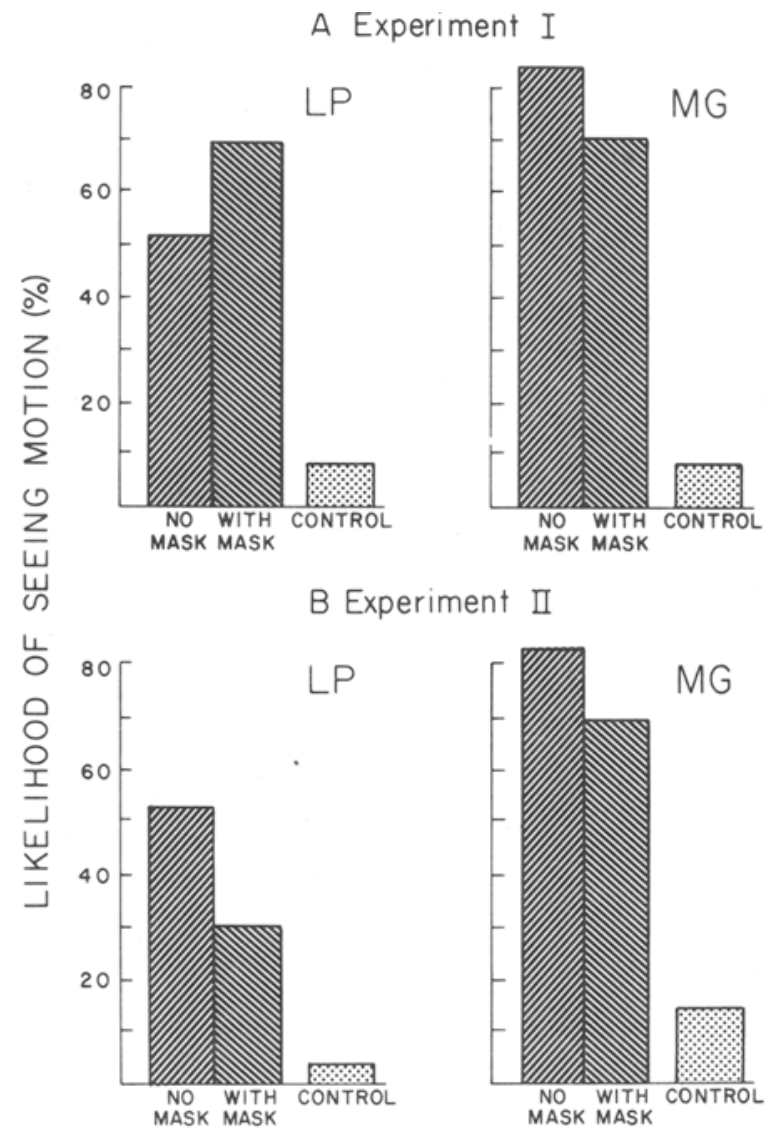

Figure 2. Likelihood of seeing smooth continuous motion at optimal SOA (S1, S2) of 100 msec for L.P. and $110 \mathrm{msec}$ for M.G. Results are compared for motion without and with masking of the first stimulus (A) or the second stimulus (B) and for a control condition (see text for detalls). 
They confirm earlier reports (von Grünau, 1978a, 1979) that motion occurred quite readily when the first bar was also optimally masked. To exclude the possibility that motion observed while the first stimulus was masked was due to motion between the mask and the second (right) bar, this type of motion was measured as a function of the interval between the mask and the second bar [SOA(mask, S2)] while the first bar was not presented. In Figure 2, the likelihood of this motion (control) is plotted for an interval corresponding to the same optimal SOA, since SOA(S1, S2) = SOA(masking) + SOA(mask, S2). It is clear that the amount of control motion was negligible for both observers. It can, therefore, be concluded that this latter type of motion did not interfere with motion between the bars when the first bar was masked at the same time (see Discussion).

The same optimal SOA (S1, S2) was used for the measurement of the orientation of the apparently moving object. In Figure 3, the orientation of the comparison stimulus is plotted as a function of the position along the motion path. Included also are control measurements $(C)$ for presentation of only the left bar or only the right bar. When there was no masking, the orientation of the apparently moving bar changed smoothly from $90 \mathrm{deg}$ (vertical) to 0 deg (horizontal). This was tested by performing analyses of variance. The orientation change was highly significant $(F=1,866, p<.00001$ for M.G.;

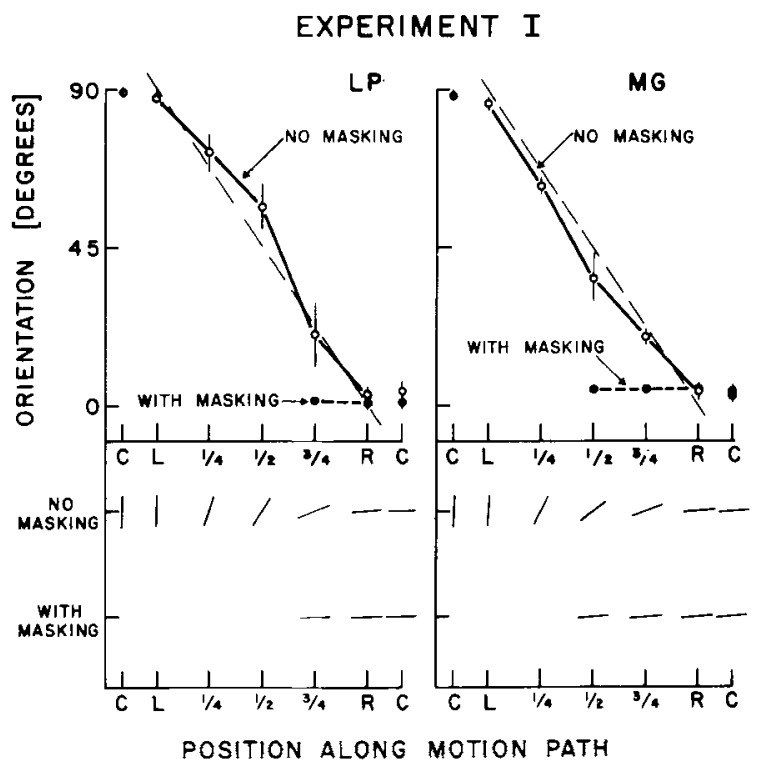

Figure 3. Orientation of the apparently moving bar at various positions along the motion path for the no-masking and withmasking conditions. $C$ is a control measure when only the respective flashed stimulus was presented. Each data point is based on 20 adjustments. Vertical lines indicate \pm 1 standard deviation. In the lower panels, the orientation of the bar is plotted directly. Results are for Experiment 1.
$F=740, p<.00001$ for L.P.). For both observers, this change was very close to being linear (interrupted line).

The situation was very different when the first bar was masked. No change of orientation could be observed. Rather, whenever the observers were able to adjust the orientation, the moving bar was seen as horizontal. It turned out that the observers were unable to make consistent judgments not only at the position of the left bar, which was not visible because it was masked, but also at the $1 / 4$ position. Here, the right bar of the mask was fairly close and seemed to interfere. Observer L.P. also had some difficulty adjusting the orientation at the $1 / 2$ position.

It is clear, however, that whenever consistent judgments could be made, the apparently moving bar always had a horizontal orientation. No influence of the vertical orientation of the masked bar could be found, in contrast with the "no-masking" condition. These results were tested within an analysis of variance and by performing a Newman-Keuls test on the means. There was a strong effect of the mask $(F=566$, $p<.00001$ for M.G.; $F=101, p<.00001$ for L.P.), and the means of the mask condition did not differ significantly ( $p<.01$ for both observers) from the mean for the horizontal orientation in the no-masking condition.

In the lower part of Figure 3, these results are presented in a more direct way. The orientation of the comparison stimulus is drawn directly, comparing the masking and no-masking conditions. Whereas the orientation changed smoothly from vertical to horizontal with no masking, only the horizontal orientation was seen with masking.

\section{Experiment 2: Masking of the Second Stimulus}

As in the first experiment, the optimal SOA value for masking of the second (right) stimulus by the mask was determined for each observer $(60 \mathrm{msec}$ for L.P., $40 \mathrm{msec}$ for M.G.). Motion between the two bars was then assessed when the second bar was not masked and when it was masked optimally by the mask. In the lower part of Figure 2, the likelihood of seeing motion is graphed for the optimal interval between the two bars for both of these conditions (100 msec for L.P., $110 \mathrm{msec}$ for M.G.). Again, motion occurred very readily when the second bar was masked, confirming earlier results (von Grünau, 1978a, 1979). In addition, control measures were taken for motion between the first bar and the mask in its rightward position, which might have interfered with the motion between the two bars. Motion between the first bar and the mask, marked as a control, was negligible.

The same optimal values of SOA (S1, S2) were used for the orientation measurements. These results are shown in Figure 4. Orientation of the comparison 
EXPERIMENT II



Figure 4. Same as Figure 3, but results are for Experiment 2.

stimulus was matched to the orientation of the apparently moving bar. Again, the smooth change that was found without masking did not occur when the second bar was masked. Rather, at all positions at which consistent measurements were possible, the apparently moving bar had the vertical orientation of the first stimulus. No consistent measurements were possible for either observer at the position of the second bar, which was masked, and at the $3 / 4$ position, where part of the mask was still fairly close and seemed to interfere. Statistical tests performed on these data (ANOVA, Newman-Keuls) confirm the strong effect of the mask $(F=2,026, p<.00001$ for M.G.; $F=259, p<.00001$ for L.P.) and show that all means in the mask condition (except the one for the $1 / 2$ position for L.P.) do not differ significantly $(p<.01)$ from the mean for the vertical orientation in the no-masking condition.

The apparently moving bar, therefore, always had a vertical orientation when the horizontal bar was masked. This is in contrast with the no-masking condition, in which the orientation changed smoothly from vertical to horizontal. These relationships are pictured directly in the lower part of Figure 4.

\section{DISCUSSION}

In the present experiments, the perceived orientation of an apparently moving bar was measured. The apparent motion occurred between two positions at which the bar had different (real) orientations.

When one of the two bars was masked in a metacontrast situation, motion could still be seen very readily, and it was usually motion of an object that was clearly defined in its shape and pattern detail. It has been suggested that metacontrast masking interferes selectively with pattern-related aspects of the masked stimulus (Breitmeyer \& Ganz, 1976). This would leave only the unmasked stimulus in the present stroboscopic-motion-with-masking situation to provide pattern information for the apparently moving object. In previous experiments (von Grünau, 1978a, 1979), however, it was not possible to determine the source of the pattern information available to the apparently moving object in this situation. Both stimuli could have contributed. The results of the present study - namely, that the masked stimulus does not seem to be able to provide pattern information that could be used by the stroboscopic motion mechanism-support the contention that metacontrast involves a selective interference with the processing of pattern information. The results also show that the metacontrast effect occurs at a point in the system that is functionally earlier than the stroboscopic motion mechanism.

It could be argued that the same kind of evidence is provided by the simple fact that, in a usual metacontrast situation, motion is seen between the target and the mask while the target is invisible. However, in those situations, it is not possible to analyze the kind of motion in any detail. Does this motion involve a moving object, and, if so, does it change shape? This cannot be assessed accurately because of the very small motion path. For this reason, the motion path has been lengthened as much as possible in the present experiments.

It is this very small distance between the target and the mask (.2 deg), as compared with the long motion path between the two bars $(5.1 \mathrm{deg})$, and the fact that the mask surrounds the target that make it very unlikely that any motion between target and mask could function as a step for the motion between the two bars. The results of the control conditions argue in the same direction. Furthermore, if the mask would have participated in the observed motion, one should have perceived a clear orientation change, since the mask had the same orientation as the target. This is exactly opposite to what was found (see Figures 3 and 4). It should be concluded, then, that the mask did not participate in the motion between the two bars, yet very effectively suppressed pattern information of the masked bar.

When the strength of apparent motion was measured (likelihood of seeing motion), the observers had to report the occurrence of smooth, continuous motion across the whole space between the two end positions (see Method). When there was masking, this meant smooth motion from or to the vicinity of the most proximal part of the mask. Motion of this kind was perceived very readily by both observers (see Fig- 
ure 2). The orientation of the moving bar, and its change when there was no masking, seemed to be quite obvious at all times. Yet, when the orientation was measured directly by adjusting a matching comparison stimulus, the observers were not able to make consistent adjustments at positions one-fourth of the path away from the masked endpoint. At these positions, adjustments were possible only very infrequently. On most occasions, however, no moving object could be seen. An explanation for this discrepancy may be found in the influence of directed attention on stroboscopic motion. Such an effect, in which the occurrence of stroboscopic motion would be reduced in regions to which attention is directed, was discussed in Kolers and von Grünau (1977). In the present case, stroboscopic motion may be weak in positions close to the masked stimulus and may, therefore, occur when attention is distributed over the whole display, but not when it is concentrated on one particular point.

While a low-level explanation in terms of pattern ("sustained") and movement ("transient") channels in the peripheral visual system is preferred here, this does not exclude the possibility of high-level explanations. Thus, an impletion process that fills in the pattern and motion details of the apparently moving object in the space between the two flashes would have to use information supplied by the two flashes. The present experiments show that some of this information is not available for whatever reason (e.g., "the first flash is not seen'), but this impletion process is flexible enough to extend the use of the stillavailable information. It is also shown that this process does not use other irrelevant information.

\section{REFERENCES}

Breitmeyer, B. G., \& Ganz, L. Implications of sustained and transient channels for theories of visual pattern masking, saccadic suppression, and information processing. Psychological Review, 1976, 83, 1-36.

Cleland, B. G., Dubin, M. W., \& Levick, W. R. Sustained and transient neurones in the cat's retina and lateral geniculate nucleus. Journal of Physiology, 1971, 217, 473-496.
Cleland, B. G., Levick, W. R., \& Sanderson, J. J. Properties of sustained and transient cells in the cat's retina. Journal of Physiology, 1973, 228, 649-680.

Dow, B. M. Functional classes of cells and their laminar distribution in monkey visual cortex. Journal of Neurophysiology, 1974, 37, 927-946.

Enroth-Cugell, C., \& Robson, J. G. The contrast sensitivity of retinal ganglion cells of the cat. Journal of Physiology, 1966, 187, 517-552.

FukUdA, Y., \& Stone, J. Retinal distribution and central projections of $\mathrm{Y}-, \mathrm{X}$ - and $\mathrm{W}$-cells of the cat's retina. Journal of Neurophysiology, 1974, 37, 749-772.

IKEDA, H., \& WRIGHT, M. J. Evidence for "sustained" and "transient" neurones in the cat's visual cortex. Vision Research, 1974, 14, 133-136.

IKEDA, H., \& WRIGHT, M. J. Spatial and temporal properties of "sustained" and "transient" neurones in area 17 of the cat's visual cortex. Experimental Brain Research, 1975, 22, 363-383.

Kole rs, P. A., \& von Grünau, M. W. Fixation and attention in apparent motion. Quarterly Journal of Experimental Psychology, 1977, 29, 389-395.

KULikowski, J. J. Pattern and movement detection in man and rabbit: Separation and comparison of occipital potentials. Vision Research, 1978, 18, 183-189. (a)

Kulikowski, J. J. Spatial resolution for the detection of pattern and movement (real and apparent). Vision Research, 1978, 18, 237-238. (b)

Kulikowski, J. J., \& Tolhurst, D. J. Psychophysical evidence for sustained and transient detectors in human vision. Journal of Physiology, 1973, 232, 149-162.

LENNIE, P. Parallel visual pathways: A review. Vision Research, 1980, 20, 561-594.

Singer, W., \& Bedworth, N. Inhibitory interaction between $\mathrm{X}$ and $\mathrm{Y}$ units in the cat lateral geniculate nucleus. Brain $R e$ search, 1973, 49, 291-307.

Tolnurst, D. J. Separate channels for the analysis of the shape and the movement of a moving visual stimulus. Journal of Physiology, 1973, 231, 385-402.

voN GrünaU, M. W. Dissociation and interaction of form and motion information in the human visual system. Vision Research, 1978, 18, 1485-1489. (a)

von Grünau, M. W. Interaction between "sustained" and "transient" channels: Form inhibits motion in the human visual system. Vision Research, 1978, 18, 197-201. (b)

von GrünaU, M. W. Form information is necessary for the perception of motion. Vision Research, 1979, 19, 839-841.

Wertheimer, M. Experimentelle Studien über das Sehen von Bewegung. Zeitschrift für Psychologie, 1912, 61, 161-265.

(Manuscript received October 14, 1980; revision accepted for publication July 13,1981 .) 